\title{
Über einen indirekten Einfluß der Sonnenaktivität auf die Intensität der kosmischen Strahlung
}

\author{
Theorie der 27-Tage-Variation, \\ Existenz und Theorie einer solaren 11-Jahre-Welle der kosmischen Strahlung
}

Von E. G. v. RokA

Aus dem Max-Planck-Institut für Physik, Göttingen

(Z. Naturforschg. 5 a, $517-530$ [1950]; eingegangen am 30. August 1950)

I. In einer kritischen Übersicht werden verschiedene Theorien der 27-Tage-Variation der kosmischen Strahlung an Hand des vorliegenden experimentellen Materials besprochen.

II. Die Messungen zeigen übereinstimmend, da $\beta$ die Sonnenfleckenrelativzahlen und die Ca-Flocken-Charakterzahlen am engsten mit der 27-Tage-Variation der kosmischen Strahlung korreliert sind. Auf Grund dieses Befundes läßt sich eine neue Theorie der 27-Tage-Variation der kosmischen Strahlung entwickeln.

Es ist bekannt, daß zwischen den Sonnenfleckenrelativzahlen bzw. den Ca-Flocken-Charakterzahlen und der solaren Ultraviolettstrahlung ein linearer Zusammenhang besteht.

Aus neuen Untersuchungen über den Entstehungsort der Mesonen folgt, daß etwa ein Drittel der Mesonen in dem oberen Teil der Ozonschicht erzeugt wird, wo infolge der Ultraviolettabsorption starke Temperaturerhöhung herrscht.

Die Temperaturschwankungen, welche durch die Variation der solaren Ultraviolettstrahlung verursacht werden, können daher entsprechend der Theorie des Temperatureffektes, durch Beeinflussung des Entstehungsortes der Mesonen, zum mindesten einen Teil der beobachteten 27-Tage-Variation der kosmischen Strahlung hervorrufen.

Die theoretische Berechnung ergibt, daß man mit durchaus plausiblen Annahmen über die Temperaturschwankung sogar die ganze 27-Tage-Variation erklären kann.

III. Die Existenz einer 11-Jahre-Schwankung der kosmischen Strahlung von mehreren Prozenten der Intensität wird in Ionisationskammerregistrierungen von Huancayo und Cheltenham nachgewiesen. Zwischen den Jahresmitteln der kosmischen Strahlung und der Sonnenfleckenrelativzahlen ergibt sich wieder eine sehr gute negative Korrelation. Der Korrelationskoeffizient beträgt $-0,91$.

Auch energiereiche Stöße $\left(4-8 \cdot 10^{10} \mathrm{eV}\right)$ zeigen starke Schwankungen, die Amplitude ist wesentlich größer als bei der allgemeinen Ionisation.

IV. Die theoretische Deutung der 11-Jahre-Periode der kosmischen Strahlung kann in analoger Weise wie bei der 27-Tage-Variation erfolgen.

Die starke Schwankung der Stöße weist - in Übereinstimmung mit andersartigen Meßergebnissen - darauf hin, daß die Stöße in der Höhe von Huancayo ( $3350 \mathrm{~m})$, wenigstens zum Teil, durch kurzlebige Teilchen mit einer Lebensdauer von etwa $10^{-8} \mathrm{sec}$ ausgelöst werden.

\section{Über die 27-Tage-Variation der kosmischen Strahlung}

I. Untersuchungen über die Ursache der 27-Tage-Variation der kosmischen Strahlung

$\mathrm{D}$ urch experimentelle Untersuchungen wurden in den letzten Jahren wesentliche neue Einzelheiten über das phänomenologische Bild der 27-Tage-Variation der kosmischen Strahlung erhalten, die eine Revision der theoretischen Vorstellungen über die Ursache der 27-Tage-Variation erforderlich machen. Im folgenden sollen zuerst die vorliegenden Theorien an Hand des experimentellen Materials besprochen werden.

\section{Deutung mit Hilfe des allgemeinen Magnetfeldes der Sonne}

In der ersten konsequenten Theorie über die Ursache der 27-Tage-Variation der kosmischen Strahlung von $\mathrm{V}$ a ll atr a und $\mathrm{G}$ o d a r ${ }^{1}$ wurde angenommen, daß die Sonne, analog zu der Erde, ein permanentes allgemeines Magnetfeld hat und die Achse des Magnetfeldes einen kleinen Winkel (etwa $6^{\circ}$ ) mit der Drehachse der Sonne bildet. Die Drehung der Sonne würde dann eine Schwankung der Intensität des

1 M. S. Vallatra u. O. Godart, Rev. Mod. Physics 11, 180 [1939]. 
Magnetfeldes in der Umgebung der Erde verursachen und damit durch Verschiebung der magnetisch erlaubten Energiegrenze für die einfallenden Primärteilchen schließlich die Intensitätsschwankungen in der kosmischen Strahlung erzeugen.

Die bei dieser Deutung sich ergebenden Schwierigkeiten sind $\mathrm{u}$. a. von $\mathrm{F}$ or bu sh eingehend erörtert worden ${ }^{2.3}$. F orbush weist darauf hin, daß nach den Rechnungen von Vallatra und Godart die Amplitude der 27-Tage-Welle auf dem Äquator praktisch verschwindend klein gegenüber der Amplitude auf $50^{\circ}$ Breite sein sollte. In Wirklichkeit war die Amplitude auf den Stationen Huancayo $\left(12^{\circ} \mathrm{S}\right)$ und Christchurch $\left(43,5^{\circ} \mathrm{S}\right)$ innerhalb der statistischen Fehlergrenze übereinstimmend. Zweitens würde eine konstante, persistente Welle zu erwarten sein. Die eingehende statistische Analyse der Registrierungen von Huancayo und Christchurch ${ }^{3}$ konnte die Existenz einer persistenten Welle nicht nachweisen *. Aus dem Vergleich der 27-Tage-Variation der kosmischen Strahlung mit der 27-Tage-Welle der erdmagnetischen Schwankungen folgert F or b u s h, daß die 27-TageVariation der kosmischen Strahlung quasipersistent sei.

Gill ${ }^{4}$ sowie Monck und Compton ${ }^{5}$ bezeichnen - allerdings aus anderen Gründen als Forbush - die 27-Tage-Variation der kosmischen Strahlung ebenfalls als quasiperiodisch.

Demgegenüber vertreten $\mathrm{Gheri}$ und $\mathrm{S}$ tein $\mathrm{maurer}$ in einer Arbeit von $1947^{6,7}$ den Standpunkt, daß mit statistischen Mitteln eine stark gestörte persistente Welle nachgewiesen werden konnte. Als wesentliches Argument für eine persistente Welle wird u. a. ein Punktwolkendiagramm angeführt ${ }^{* *}$. Die „Expektanz“ wurde hierbei aus Streuung der Tagesmitteln mit Hilfe einer Formel berechnet, die für zufällige Verteilung gilt ${ }^{* * *}$. Die Amplitude des

2 S. E. F o r bus h, Rev. Mod. Physics 11, 190 [1939].

3 S. E. F o r b u s h, Bull. Int. Union. Geod. and Geophysik No. 11, 438 [1940].

4 P. S. Gill, Physic. Rev. 55, 429 [1939].

5 A. T. Monck u. A. H. Compton, Rèv. Mod. Physics 11, 173 [1939].

6 H. Gheri u. R. S t e in m a urer, Terrest. Magn. atm. Elektr. 52, 343 [1947].

7 H. Gheri u. R. S tein ma urer, Acta physica Austriaca 1, 42 [1947].

8 S. Chapman u. J. B a rtels, Geomagnetism,

2. Bd., Clarendon Press, Oxford 1940.

9 K. S t u m p f f, Periodenforschung, J. Springer, Ber$\operatorname{lin} 1937$.

10 G. T h i e s s e n, Z. Astrophysik 26, 16 [1949].

11 J. W. B r o x o n, Physic. Rev. 70, 494 [1946].

12 J. W. B r ox o n, Physic. Rev. 72, 525 [1947].
Schwerpunktes beträgt die dreifache Schwerpunktsexpektanz. Hieraus kann aber nur gefolgert werden, daß eine Wiederholungstendenz vorhanden ist. Die Unterscheidung zwischen persistenten und quasipersistenten Charakter müßte dann noch besonders durchgeführt werden. Analog verhält es sich mit den anderen Argumenten, die für die Persistenz gebracht werden; sie zeigen zwar, daß eine deutliche Wiederholungstendenz vorhanden ist, doch beweisen sie nicht, daß eine echte Persistenz und nicht nur eine Quasipersistenz vorliegt.

Es ergibt sich also, daß der für die VallatraGodartsche Theorie der 27-Tage-Variation der kosmischen Strahlung wesentliche Nachweis einer persistenten Welle bisher noch nicht erbracht worden ist.

Das Problem kann wohl nur durch Bearbeitung einer längeren Meßreihe wie bei den bisherigen Veröffentlichungen (F or b u s h 34, G heri und S tein maurer 30 Sonnenrotationen) entschieden werden.

Neuerdings wird die Existenz eines allgemeinen Magnetfeldes der Sonne von der Stärke, wie für die Erklärung der 27-Tage-Variation der kosmischen Strahlung erforderlich wäre, von der astrophysikalischen Seite - auf Grund neuerer genauerer Messungen - überhaupt angezweifelt ${ }^{10}$.

Sehr wichtige Ergebnisse lieferten in den letzten Jahren die Untersuchungen von W. Broxon ${ }^{11-13}$, die sich ebenfalls recht wenig mit einer sonnenmagnetischen Theorie vereinbaren lassen. $\mathrm{Br}$ ox o $\mathrm{n}$ fand, daß auch die kleinen Stöße in einer Ionisationskammer hinter $50 \mathrm{~cm} \mathrm{Pb-Äquivalent} \mathrm{eine} \mathrm{27-Tage-}$ Variation zeigen und die Amplitude sogar etwa 15-fach größer ist als bei der 27-Tage-Variation der allgemeinen Ionisation. Ferner ergab sich, daß auch die Amplitude der 27-Tage-Variation der allgemeinen Ionisation hinter $50 \mathrm{~cm}$ Pb-Äquivalent $\left(0,2 \%{ }^{14}\right)$ praktisch dieselbe ist wie hinter $12 \mathrm{~cm} \mathrm{Pb-Äquivalent}$

13 J. W. B r ox o n, Physic. Rev. 72, 1187 [1947].

14 J. W. B r o x o n, Physic. Rev. 59, 773 [1941].

* Genauer: Auf S. 445 sagt Forbush: „... with the limited data available it is not possible to discriminate, by means of the characteristic, between a persistent wave and quasi-persistence." Die erhaltenen Rechnungsergebnisse über die Charakteristik werden nicht mitgeteilt. In der Zusammenfassung schreibt Forbush: .No evidence is found for the existence of a persistent 27 -day wave in cosmic-ray intensity such as would be expected if the solar magnetic moment were sufficiently large and sufficiently inclined to the Sun's axis of rotation."

** Ausführliche Literatur über die verschiedenen hier zur Erwähnung kommenden statistischen Methoden: 8, 9.

*** Üblicherweise wird die Quadratwurzel aus dem Mittelwert der Amplitudenquadrate als Expektanz bezeichnet. 
$\left(0,18 \%{ }^{4}\right)$. Es erscheint deshalb wenig wahrscheinlich, daß die 27-Tage-Variation der kosmischen Strahlung durch einen Mechanismus zustande kommt, der dadurch wirkt, daß er das weiche Ende des Spektrums abschneidet.

\section{Deutung der 27-Tage-Variation}

der kosmischen Strahlung als eine Folge der erdmagnetischen Schwankungen

Eine andersartige Theorie der 27-Tage-Variation der kosmischen Strahlung schlug F o r b u s h vor ${ }^{2,3}$. Er führt die 27-Tage-Variation der kosmischen Strahlung auf die 27-Tage-Variation des erdmagnetischen Feldes zurück. Entsprechend der Störmerschen Theorie nimmt Forbush an, daß ein Ringstromsystem im Abstand vom mehrfachen Erdradius um die Erde existiert. Die Störungen in diesem Ringstromsystem verursachen Magnetfeldschwankungen in dem Weltraum, die gegebenenfalls auf der Erde nur teilweise oder überhaupt nicht zu beobachten sind. Die Variationen des Stromsystems werden durch die Variationen in der Korpuskularstrahlung der Sonne hervorgerufen.

F orbush stützte sich bei der Aufstellung dieser Theorie weitgehend auf eine, vorher schon kurz erwähnte, statistische Untersuchung aus dem Jahre $1939^{3}$. An Hand der Ionisationskammerregistrierungen während 34 Sonnenrotationen bei Huancayo wurde bei dieser Untersuchung u. a. gezeigt, mit Hilfe eines besonderen Kunstgriffes von Bartels, daß die 27-TageWelle der kosmischen Strahlung und der amerikanischen magnetischen Charakterzahlen meistens entgegengesetzte Phase haben, wobei der gefundene Phasenunterschied von etwa $180^{\circ}$ sicher nicht durch Zufall vorgetäuscht wurde.

F o r b u s h folgert hieraus: 1 . Weil die amerikanischen Charakterzahlen bekanntlich mit den internationalen magnetischen Charakterzahlen eng korreliert sind und die letzteren eine quasipersistente Welle haben, die 27-Tage-Welle der kosmischen Strahlung ebenfalls quasipersistent ist. 2. Die 27Tage-Welle der kosmischen Strahlung wird durch Störungen des erdmagnetischen Feldes erzeugt.

So bestechend auch im ersten Moment diese Schlußfolgerung erscheint, kann sie doch bei eingehender Betrachtung nicht als zwingend bezeichnet werden. Die negative Korrelation zwischen den magnetischen Charakterzahlen und der 27-Tage-Variation der kosmischen Strahlung kann z. B. auch dadurch zustande kommen, daß die 27-Tage-Variation durch irgendein anderes mit der Sonnentätigkeit zusammenhängendes Phänomen erzeugt wird, das mehr oder weniger stark mit der Korpuskularstrahlung der Sonne korreliert ist. So weiß man z. B., daß die Monatsmittel der magnetischen Charakterzahlen und der Sonnenfleckenrelativzahlen einen Korrelationskoeffizient von $+0,65$ zeigen ${ }^{15}$. Andererseits hat z. B. auch die Ultraviolettstrahlung der Sonne eine sehr gute Korrelation mit den Sonnenfleckenrelativzahlen. Man muß also noch eine weitergehende Untersuchung führen, um einen ursächlichen Zusammenhang zwischen der 27-TageVariation der kosmischen Strahlung und den magnetischen Charakterzahlen zu beweisen. Man kann hierbei - wenn man mit statistischen Mitteln arbeiten will - etwa 1. die Persistenz beider Wellen untersuchen oder 2. die Korrelation mit verschiedenen solaren Elementen zum Vergleich heranziehen. F or bush selbst macht hierüber keine Angaben. Von anderen Verfassern liegen jedoch Arbeiten über dieses Problem vor und zeigen übereinstimmend, daß die erdmagnetischen Störungen nicht die Ursache der 27-Tage-Variation der kosmischen Strahlung sein können.

M onck und Compton haben in ihren Untersuchungen über die 27-Tage-Variation der kosmischen Strahlung ${ }^{5}$ die Wiederholungstendenz der kosmischen Strahlung mit der Wiederholungstendenz der magnetischen Charakterzahlen verglichen. Sie verwendeten die $\mathrm{Chr}$ e esche Methode. Es zeigte sich, daß die sekundären Pulse der kosmischen Strahlung in dem betrachteten Intervall (4 Pulse vor und nach den Primären) in ihrer Amplitude im Gegensatz zu der 27-Tage-Variation der magnetischen Charakterzahlen keine Abnahme zeigen. Auch die Kurvenform für die magnetischen Charakterzahlen und die kosmische Strahlung sind verschieden. Aus beiden Tatsachen folgern die Verfasser: „The magnetic and cosmic-ray disturbances of 27-day-period are of different origin“.

In einer späteren Untersuchung fanden $\mathrm{M}$ o n c $\mathrm{k}$ und Compton - nach der Mitteilung von B rox o $\mathrm{n}^{14}$-, daß sogar bis zu den zehnten Pulsen eine persistente Amplitude vorhanden war, während sich bei der ersten Ausdehnung der Untersuchung bis zu dem achten Puls zuerst eine Abnahme zeigte ${ }^{\mathbf{1 6}}$.

Der Befund von Monck und Compton über die guten Persistenzen der kosmischen Strahlung konnte von $\mathrm{Broxon}$ bestätigt werden ${ }^{14}$. Er fand ebenfalls bei seiner Untersuchung mit Hilfe der

15 J. B a rtel s, Terrest. Magn. atm. Elektr. 37, 40 [1932].

16 A. H. Compton u. A. T. Monck, Physic. Rev. 59, 112 [1941]. 
Chreeschen Methode bis zu den vierten Pulsen, daß die Pulse noch keine Abnahme in der Amplitude zeigten.

Kolhörster hat systematisch verschiedene erdmagnetische und solare Elemente mit der kosmischen Strahlung verglichen, die während der Sonnenrotationen 1433-1439 (Zählung nach B a r tels) gemessen wurden ${ }^{17}$. Der Korrelationskoeffizient betrug mit der magnetischen Kennziffer - 0,31, mit der horizontalen (Nord-Süd-[X-]) und der vertikalen (Z-)Komponente des Erdmagnetismus $+0,65$ bzw. $-0,52$. Noch besser waren die Korrelationskoeffizienten mit den Sonnenfleckenrelativzahlen (ganze Scheibe) - 0,78 und mit den Ca-Flocken (ganze Scheibe) - 0,82.

Nach Kolhörster sinkt die Intensität der kosmischen Strahlung „etwa in dem Maße, wie die aktiven Zentren vom Sonnenrand zum Meridian vorrücken. Umgekehrt steigt sie dann bis zum Verschwinden der Zentren hinter dem Sonnenrand wieder an“.

Bei dem Vergleich der 27-Tage-Variation der kosmischen Strahlung mit den magnetischen Störungen sagt Kolhörster : „Ein ähnliches Verhalten ist ja von den erdmagnetischen Variationen her bekannt. Diesen gegenüber dürfte jedoch ein wesentlicher Unterschied darin zu suchen sein, daß die erdmagnetischen Störungen viel unvermittelter einsetzen, während die Intensitätswerte der Höhenstrahlung im großen und ganzen sich mehr kontinuierlich ändern."

Die wichtigste Schlußfolgerung der Kolhörsterschen Arbeit: „Ganz allgemein erweisen sich also Flocken bisher am engsten mit der Intensität der Höhenstrahlung korreliert.“

B rox on untersucht mit Hilfe der Chreeschen Methode die Korrelation zwischen kosmischer Strahlung, internationalen magnetischen Charakterzahlen und Sonnenfleckenflächen ${ }^{18}$. Während bei primären Pulsen allgemein noch eine entgegengesetzte Phase zwischen kosmischer Strahlung und magnetischen Charakterzahlen vorhanden war, zeigte sich bei den sekundären Pulsen kein erkennbarer Zusammenhang. „The curves do not provide any clear indication of magnetic pulses corresponding to the secondary cosmic-ray pulses.“ „Rather surprisingly, the secondary sunspot-area pulses . . . are in general larger and

17 W. K olhörster, Physik. Z. 40, 107 [1939].

18 J. W. B r ox on, Physic. Rev. 62, 508 [1942].

19 J. Z ir k l e r, Z. Naturforschg. 4 a, 394 [1949].

20 S. E. F or bus h, Physic. Rev. 70, 771 [1946].

20a A. E h m e r t, Z. Naturforschg. 3a, 264 [1948].

21 E. B a g g e , Ergebn. exakt. Naturwiss. XXII, 202 [1949]. more clearly defined in relation to the primary" (cosmic-ray pulses) ,than are the corresponding secondary magnetic pulses".

Die. Korrelation zwischen Häufigkeit der kleinen Stöße, magnetischen Charakterzahlen und Sonnenfleckenflächen wurden von J. W. B r o x o n mit Hilfe der Chree-Methode ebenfalls untersucht ${ }^{12,13}$. „The method does not indicate clearly a dependence of burst frequency upon magnetic character.“* „It does strongly indicate a relation to sunspot areas."

Im gesamten folgt also, daß die Schwankung des erdmagnetischen Feldes schon aus statistischen Gründen, wegen der verschiedenen Persistenz und schlechteren Korrelation im Vergleich mit verschiedenen solaren Elementen offenbar nicht allein die Ursache der 27-Tage-Variation der kosmischen Strahlung sein kann.

Gegen diese statistischen Argumente kann noch der Einwand erhoben werden, daß die vorhandene schlechte Korrelation dadurch zustande kommt, daß die Änderungen in dem Störmerschen Ringstromsystem an der Erde nicht beobachtet werden können. Damit entgleitet die Theorie von F or b u s h der statistischen Prüfbarkeit.

Doch bleibt auch in diesem Fall bestehen, daß die 27-Tage-Variation der kleinen Stöße mit ihrer 15-fach größeren Amplitude als die allgemeine Ionisation allen Theorien - auch der erdmagnetischen Theorie -, die durch das Abschneiden des weichen Endes des Spektrums die 27-Tage-Variation erklären wollen, widerspricht.

\section{Die Annahme einer solaren. Komponente} als Ursache der 27-Tage-Variation der kosmischen Strahlung

Nachdem Zirkler ${ }^{19}$ Andeutungen gefunden hatte und Forbush20 und Ehmert ${ }^{20 a}$ überzeugend nachweisen konnten, daß solare Eruptionen zeitweise von plötzlichen Intensitätszunahmen der kosmischen Strahlung begleitet sind, wurde auch die Vermutung geäußert, daß die 27-Tage-Variation der kosmischen Strahlung durch eine solare Komponente der kosmischen Strahlung erzeugt wird ${ }^{\mathbf{2 1}}$.

Bei dieser Deutung tritt die Schwierigkeit auf, daß, obwohl die kosmische Strahlung bei Huancayo - im Gegensatz zu den nichtäquatorialen Stationen keine solare Intensitätszunahmen zeigt ${ }^{20}$, in Huan-

* Mit Rücksicht auf die großen Energien, die die stoßauslösenden Primärteilchen haben müssen, ist natürlich von vornherein eine magnetische Beeinflussung unwahrscheinlich. 
cayo eine sehr deutliche 27-Tage-Variation vorhanden ist.

Ferner wäre bei der Annahme einer solaren Komponente als Ursache der 27-Tage-Variation der kosmischen Strahlung eine positive Korrelation zwischen Sonnenfleckentätigkeit und kosmischer Strahlung zu erwarten. Mit zunehmender Aktivität der Sonne sollte die Intensität der kosmischen Strahlung ebenfalls zunehmen.

Positive Korrelation zwischen Sonnentätigkeit und kosmischer Strahlung wurde bisher nur bei der Verwendung von $\mathrm{H} a$-Flocken-Charakterzahlen als Maßzahl für die Sonnenaktivität ${ }^{22}$ und bei einem paradoxen Ergebnis von B r ox on ${ }^{18}$ unter Verwendung von Sonnenfleckenflächen gefunden. Beide Male war die Realität der gefundenen Korrelation nicht gesichert.

Nach den Untersuchungen von $\mathrm{Miczaika}{ }^{23}$ stehen die $\mathrm{H} \alpha$-Flocken-Charakterzahlen - im Gegensatz zu den Sonnenfleckenrelativzahlen und CaFlocken-Charakterzahlen - mit der kosmischen Strahlung nicht im Zusammenhang.

B rox o n hat bei seinen Untersuchungen über die 27 - Tage-Korrelation der kosmischen Strahlung ${ }^{18}$ neben der Chree-Methode auch ein zweites Verfahren angewandt. Hier wurde das Material entsprechend der untersuchten Periodenlänge in Zeilen unterteilt und jeweils aus den entsprechenden Tagen, spaltenweise, die Mittelwerte gebildet. Wenn als Periodenlänge 27 Tage gewählt wurde, zeigte die Kurve der kosmischen Strahlung und Sonnenfleckenflächen positive Korrelation. Ein sehr merkwürdiges Ergebnis - wie auch Broxon betont - , u. a. deshalb, weil das gleiche Material, untersucht mit der statistisch zuverlässigeren Chree-Methode, ein entgegengesetztes Ergebnis liefert. Nach der Chree-Methode besteht 1. zwischen kosmischer Strahlung und Sonnenfleckenflächen eine Phasenverschiebung von etwa 4-5 Tagen und 2. große Sonnenfleckenflächen sind mit niedriger Intensität der kosmischen Strahlung, kleine Flächenwerte mit erhöhter Intensität verbunden, d.h. die Chree-Methode zeigt eine negative Korrelation.

B r o x o n weist ferner darauf hin, daß die Sonnen-

22 A. Corlin, Ann. Observat. Lund 4, A 82 [1934].

23 G. R. M i c z a i k a, Z. Astrophysik 18, 146 [1939].

$24 \mathrm{H}$. Th. G r a z i a d e i, S.-B. Akad. Wiss. Wien, Abt. II a 145, 495 [1936].

25 V. F. H e ß, Terr. Magn. atm. Elektr. 41, 345 [1936].

* Auch die Tageswerte zeigen eine gute negative Korrelation. Die Untersuchung über die täglichen Werte soll an einer anderen Stelle veröffentlicht werden. fleckenkurve, die bei der 27-Tage-Mittlung erhalten wurde, nicht nur gegenüber der kosmischen Strahlung, sondern auch im Vergleich mit der Kurve der erdmagnetischen Charakterzahlen einen ungewöhnlichen Verlauf hat: „. . . comparsion . . . shows an inverse relation between sunspot and terrestrial magnetic activity in direct opposition to long accepted relations between these variables."

Es scheint also, daß man dem Ergebnis der zweiten, weniger zuverlässigen statistischen Methode, das über ein an und für sich nicht sehr langes Intervall von $1^{1} / 2$ Jahren erhalten wurde, kein besonders großes Vertrauen schenken darf.

Die Untersuchungen von anderen Verfassern, Graziadei ${ }^{24,25}$, Gheri und Steinmaurer ${ }^{6,7}$, Kolhörster ${ }^{17}$, Miczaika ${ }^{23}$, zeigen durchweg, daß zwischen der Sonnentätigkeit und Intensität der kosmischen Strahlung eine negative Korrelation besteht.

In dem zweiten Teil dieser Arbeit werden wir an Hand von Dreimonats- und Jahresmitteln ebenfalls nachweisen, daß zwischen der kosmischen Strahlung und den Sonnenfleckenrelativzahlen eine sehr gute negative Korrelation herrscht*. In den Jahren des Sonnenfleckenminimums, wo während langer Zeitspannen überhaupt kein Sonnenfleck vorhanden ist, erreicht die kosmische Strahlung ihre höchste Intensität.

\section{Eine neue theoretische Deutung}

1. Korrelation zwischen Ca-Flocken, Sonnenfleckenrelativzahlen und Intensität der Ultraviolettstrahlung der Sonne

Unsere Überlegungen über die Ursache der 27-tägigen Quasiperiode der kosmischen Strahlung nahmen ihren Ausgang von der Tatsache, daß von sämtlichen untersuchten Elementen die Sonnentätigkeit, ausgedrückt in Charakterzahlen der Ca-Flocken bzw. in Sonnenfleckenrelativzahlen, die beste Korrelation zeigt ${ }^{* *, * * *}$.

Zwischen den Ca-Flocken-Charakterzahlen bzw. den Sonnenfleckenrelativzahlen und der Ultraviolettstrahlung der Sonne besteht eine enge Beziehung.

* Die Ca-Flocken-Charakterzahlen und die Sonnenfleckenrelativzahlen sind bekanntlich miteinander eng korreliert, wobei die Korrelation um so besser, je länger die Mittelwerte sind, d. h. für Jahres- oder Monatsmittel besser als für Tagesmittel. .

$* * *$ Bei Tageswerten scheint die kosmische Strahlung mit Ca-Flocken-Charakterzahlen noch etwas bessere Korrelation als mit Sonnenfleckenrelativzahlen zu zeigen $\mathbf{2 4}, \mathbf{1 7}, \mathbf{2 3}$. 
Die Schwankung der solaren Ultraviolettstrahlung läßt sich aus der täglichen magnetischen Variation ${ }^{26-28}$ und aus der Ionisation der verschiedenen ionosphärischen Schichten berechnen ${ }^{29-33}$. Die Untersuchungen zeigen übereinstimmend, daß die Ultraviolettstrahlung in dem untersuchten Bereich aus einem konstanten und einem den Sonnenfleckenrelativzahlen proportionalen veränderlichen Anteil besteht. Dabei ist die Intensität des veränderlichen Anteils bei der Sonnenfleckenrelativzahl $\mathrm{R}=100$ etwa dieselbe wie die des konstanten Anteils.

Die Korrelation besteht, wie Bartels ${ }^{27}, 28$ und $\mathrm{K}$ i e p e $\mathrm{n}$ h e u e r ${ }^{31-33}$ mit Hilfe von Sonnenfleckenrelativzahlen und A 11 e $\mathrm{n}^{30}$ mit Hilfe von Ca-Flokken zeigen konnten, auch für die täglichen Werte.

Über die Wellenlängen der mit den Relativzahlen korrelierten, ionosphärisch und erdmagnetisch wirksamen Ultraviolettstrahlung sind nur theoretische Werte bekannt. Der Bereich des ionosphärisch wirksamen Anteiles liegt wahrscheinlich unterhalb von $900 \AA ̊$ E. Die größte Wellenlänge dürfte der erdmagnetisch wirksamen Komponente zukommen.

J. B a r t e l s sagt von der Natur dieser Strahlung ${ }^{27}$ : „Wahrscheinlich eine solare Strahlung, die ziemlich niedrig in der Ionosphäre, in oder nahe der Schicht absorbiert wird, welche von der extremen UltraviolettEmission bei einer Sonneneruption ionisiert wird.“

Andererseits schätzten vor einiger Zeit $\mathrm{Chap}$ $m$ an und Price ${ }^{34}$ die Wellenlänge der Mögel-Dellinger-Effekt * erzeugende Strahlung auf 1215,7 ÅE $(\mathrm{L} \alpha)$. Neuere Arbeiten ${ }^{35}$ weisen auf noch größere Wellenlängen hin, bis zu $2400 \AA ̊ \mathrm{E}$.

Die Wellenlänge der Ultraviolettkomponente, die das atmosphärische Ozon erzeugt, liegt unterhalb $1800 \AA ̊ \mathrm{E}$.

\section{Anteil der Mesonen, die in dem oberen Teil der Ozonschicht entstehen}

Nach unseren gegenwärtigen Kenntnissen werden die Mesonen durch aus dem Weltraum einfallende

26 J. B a rte ls, Abh. preuß. Akad. Wiss. Berlin, physik.-math. Kl., Nr. 12 [1941].

27 J. B a rte l s, Terr. Mag. atm. Elektr. 51, 181 [1946].

28 J. B a rtels, Geophysik I, S. 39; Naturwiss. u. Medizin in Deutschland 1939-1946 (FIAT-Review), Dieterich'sche Verlagsbuchhandlung, Wiesbaden 1948.

29 M. Wald meier, Helv. physica Acta 17, 168 [1944].

30 C. W. Alle n, Terr. Magn. atm. Elektr. 51, 1 [1946].

31 K. O. Kiepenheuer, Ann. Astrophysik [Paris] 8, 210 [1945].

3. K. O. Ki penheuer, Neue Physik. Bl. 1, 225 [1946].

* Der Mögel-Dehlinger-Effekt entsteht durch Veränderungen in den Schichten zwischen 60 und $90 \mathrm{~km}$ Höhe.
Primärteilchen, überwiegend Protonen, beim Eindringen in die Atmosphäre durch Wechselwirkung mit den Stickstoff- und Sauerstoffatomkernen erzeugt. Entsprechend neueren Untersuchungen entstehen zunächst $\pi$-Mesonen, die nach einer Lebensdauer von $\sim 10^{-8} \mathrm{sec}$ in $\mu$-Mesonen übergehen. Die $\pi$-Mesonen haben eine größere Bremsung infolge der Wechselwirkung und den Kernen, während die $\mu$-Mesonen im wesentlichen nur durch Ionisation Energie verlieren ${ }^{* *}$. Ferner tritt bei $\pi \rightarrow \mu$-Zerfall ein Energieverlust auf, weil etwa $30 \%$ der ursprünglichen Energie auf das bei dem Zerfall ausgesandte Neutrino übertragen wird ${ }^{36}$.

Wegen der sehr geringen Lebensdauer der $\pi^{-}$ Mesonen wird auch ihr Zerfallsweg - mit Ausnahme extrem großer Energien - ebenfalls sehr kurz, von der Größenordnung von einigen hundert Metern sein. Der Energieverlust der $\pi$-Mesonen auf dieser Strecke wird - bei den niedrigen Drucken in ihrer Entstehungshöhe - sehr gering im Vergleich mit dem Energieverlust, der zum Erreichen der Seehöhe (für $\mu$-Mesonen) erforderlich ist. Es kann deshalb für die folgende Rechnung angenommen werden, als ob unmittelbar $\mu$-Mesonen entstehen würden. (Der Energieverlust beim Zerfall wird in dem Primärspektrum der $\mu$-Mesonen berücksichtigt.) Damit können wir uns im folgenden auf die schon bekannten theoretischen Rechnungen stützen, die noch vor der Entstehung der $\pi$-Mesonen aufgestellt wurden und eine einheitliche Mesonenkomponente voraussetzen ${ }^{37-39}$.

Der Wirkungsquerschnitt der Erzeugung von Mesonen wird auf Grund von theoretischen Beobachtungen und experimentellen Arbeiten etwa gleich dem geometrischen Wirkungsquerschnitt geschätzt.

Mit dem aus der Kernphysik bekannten geometrischen Kernradius für Stickstoff $r \approx 3,3 \cdot 10^{-13} \mathrm{~cm}$ folgt dann für die Primärteilchen eine mittlere freie

${ }_{33}$ K. O. Ki e penheuer, in: P. ten Bruggenc a te, Astronomie, Astrophysik u. Kosmogonie, S. 229; Naturwiss. u. Medizin in Deutschland 1939-1946 (FIATReview). Dieterich'sche Verlagsbuchhandlung, Wiesbaden 1948.

34 S. Cha pman u. W. C. Pric e, Rep. Progr. Physics 3, 61 [1947].

$35 \mathrm{M}$ m e. u. M.E. V a s s y, Sixième Rapport de la Commission Pour L'Etude des Rélations Entre les Phénomènes Solaires et Terrestres, Orléans 1948, S. 90.

36 W. H e i s e n b e r g, Z. Physik 126, 569 [1949].

37 R. P. Feym ann u. H. A. B e the, Physic. Rev. 70, 786 [1946].

38 L. Jánossy, Cosmic Rays. Clarendon, Oxford 1948. 39 H. A. B e th e, Physic. Rev. 70, 787 [1946].

** Der Einfluß der unterschiedlichen Ionisation infolge der verschiedenen Massen ist gegenüber diesem Effekt bedeutungslos. 
Weglänge $l \approx 53 \mathrm{~g} / \mathrm{cm}$ und als mittlere Entstehungshöhe der Mesonen etwa $25 \mathrm{~mm} \mathrm{Hg}$.

Entsprechend der experimentellen Absorption der Primärteilchen ergibt sich ferner bei der Annahme von vertikalem Einfall, daß etwa $1 / 3$ der Mesonen oberhalb $25 \mathrm{~km}$ Höhe $\left(\approx 25 \mathrm{~cm} \mathrm{H}_{2} \mathrm{O}\right)$ erzeugt wird $\left(J_{0} e^{-25 / 53}=0,627\right)$.

$\mathrm{V}$ an Allen und $\mathrm{T}_{\text {ate }}{ }^{40}$ fanden in V 2 -Messungen, daß die Intensität der kosmischen Strahlung bei allseitigem Einfall oberhalb $55 \mathrm{~km}$ Höhe bis zu der erreichten größten Höhe von 120 km konstant ist, und folgern hieraus, daß die gemessenen Teilchen oberhalb $55 \mathrm{~km}$ die primären sind und etwa von dieser Grenze ab die Wechselwirkung mit den Luftmolekülen stattfindet.

Für die weiteren Betrachtungen ist die Folgerung wesentlich, daß ein bedeutender Anteil der Mesonen (etwa $1 / 3$ ) oberhalb $25 \mathrm{~km}$ und unterhalb $50 \mathrm{~km}$ Höhe entsteht.

\section{Wirkungsweise der Ultraviolettstrahlung der Sonne auf die Intensität der harten Komponente der kos- mischen Strahlung}

Wie es gut bekannt ist und neuerdings durch V2Messungen auch unmittelbar bestätigt wurde ${ }^{41}$, nimmt die Temperatur der Atmosphäre im oberen Teil der Ozonschicht - zwischen 25 und $50 \mathrm{~km}$ Höhe - infolge der Ultraviolettabsorption des Ozons stark zu. Während die Temperatur in $25 \mathrm{~km}$ Höhe (bei $50^{\circ}$ nördl. Breite) ungefähr $220^{\circ} \mathrm{K}$ ist, beträgt sie in $50 \mathrm{~km}$ Höhe nach den Berechnungen von G o w a n ${ }^{42}$ etwa $430^{\circ} \mathrm{K}$. Der genaue Verlauf hängt u. a. von der Jahreszeit ab.

Es ist deshalb sehr naheliegend, anzunehmen, daß entsprechend der zu den Sonnenfleckenrelativzahlen proportionalen Schwankungen der Intensität der Ultraviolettstrahlung im oberen Teil der Ozonschicht Temperaturschwankungen auftreten. Die damit verbundene Verschiebung der Isobaren bzw. Veränderung der Laufstrecke der Mesonen erscheint dann als Intensitätsschwankung der harten Komponente in Seehöhe.

Wir interpretieren also die 27-Tage-Quasiperiode der kosmischen Strahlung als Temperatureffekt im oberen Teil der Ozonschicht, erzeugt durch Variation der Intensität der solaren Ultraviolettstrahlung.

40 T. A. van Allen u. H. E. T at el, Physic. Rev. 73, 245 [1947].

41 N. B est, R. Havens u. H. La Gow, Physic. Rev. 71, 215 [1947].
Es sei hier bemerkt, daß der sichtbare Teil der Sonnenstrahlung keine systematische Korrelation mit den Sonnenfleckenrelativzahlen zeigt.

Die Temperaturschwankung in der oberen Ozonschicht kann durch Variation der Ozonerzeugung und durch Schwankung der absorbierten Strahlung entstehen.

Systematische Temperaturmessungen oder Bestimmungen der Isobarlinien in den Schichten zwischen 25 und $50 \mathrm{~km}$ Höhe liegen, soweit wir übersehen konnten, noch nicht vor.

\section{Theoretische Berechnung der 27-Tage-Variation}

Das Ziel der theoretischen Berechnung war, die Variation der Mesonenintensität in Nähe der Meereshöhe zu berechnen, die durch eine Temperaturschwankung in der oberen Ozonschicht verursacht wird, und festzustellen, ob die vorausgesetzte Temperaturschwankung durch Variation der solaren Ultraviolettstrahlung hervorgerufen sein kann.

Das zugrunde gelegte physikalische Bild entsprach den üblichen Annahmen 43, 38, 37. Die aus dem Weltraum einfallenden Primärteilchen werden, während sie Mesonen erzeugen, exponentiell absorbiert. Der Verlauf der Mesonenintensität im weiteren wird dann durch Ionisation und Zerfall bestimmt.

Der Impulsverlust infolge Ionisation wurde für alle Mesonen, unabhängig von ihrer Energie, zu

$$
a=2 \cdot 10^{6} \mathrm{eV} / c \text { per } \mathrm{cm} \mathrm{H}_{2} \mathrm{O}
$$

angenommen.

Die Wahrscheinlichkeit, daß ein Meson von der Masse $\mu$ und mittleren Lebensdauer $\tau$ (in Eigenzeit), erzeugt in der Atmosphäre an der Stelle $\sigma_{e}$, den Beobachtungsort $\sigma_{B}$ mit dem Impuls $p$ erreicht, ergibt sich dann zu

$w=\exp -p_{\tau} \int_{\sigma_{e}}^{\sigma^{B}} \frac{t}{(p+q-a \sigma) \sigma} d \sigma$,

wobei

$$
p={ }_{\tau}^{\mu} H \quad \text { und } \quad t=\frac{T}{273}
$$

bezeichnet. $T$ ist die absolute Temperatur der Atmosphäre an der Stelle, wo der Druck $\sigma \mathrm{cm}$ Wassersäule ist. Ferner $q-a \sigma=a\left(\sigma_{B}-\sigma\right)$ ist der Impulsverlust zwischen dieser Stelle und dem Beobachtungsort.

42 E. H. G ow a n, Proc. Roy. Soc. [London], Ser. A 190, 219 [1947].

${ }^{43}$ L. János s y u. J. G. Wils on, Nature [London] 158,450 [1946]. 
$H=7,98 \mathrm{~km}$ ist die Höhe der homogenen Atmosphäre von $0^{\circ} \mathrm{C}$.

Das differentielle Mesonenspektrum an der Beobachtungsstelle $\sigma_{B}$ wird

$$
S(p)=\frac{S_{0}}{l} \int_{0}^{\sigma_{B}} e^{-\frac{\sigma_{e}}{l}-p_{\tau} \int_{\sigma_{e}}^{\sigma_{B}} \frac{t}{(p+q-a \sigma) \sigma} d \sigma} d \sigma_{e},
$$

wobei $S_{0}$ das differentielle Mesonenspektrum am Entstehungsort, $l$ die mittlere freie Weglänge der Primärteilchen ist.

Für den Spezialfall einer isothermen Atmosphäre $(t=1)$ führt diese Gleichung zu dem von Já n o s s y und Wils on gegebenen Ausdruck ${ }^{43}$, und hieraus wieder ergibt sich mit der vereinfachenden Annahme, $\mathrm{da} ß$ die Mesonen in gleicher Höhe erzeugt werden, die bekannte Formel von $\mathrm{Euler}$ und $\mathrm{H}$ e is enb e r g ${ }^{44}$.

Wenn die Temperatur der Atmosphäre um $t=f(\sigma)$ variiert, ändert sich die Intensität der Mesonen entspr. Gl. (2) um den Betrag

$$
\jmath S=\frac{S_{0}}{l} \int_{0}^{\sigma_{B}} e^{F} \delta F d \sigma_{e},
$$

hierbei

$$
\begin{aligned}
& F=-\frac{\sigma_{e}}{l}-p_{\tau} \int_{\sigma_{e}}^{\sigma_{B}}(p+q-a \sigma) \sigma \\
& \text { und } \delta F=-p_{\tau} \int_{\sigma_{e}}^{\sigma_{B}} \frac{\delta t}{(p+q-a \sigma) \sigma} d \sigma
\end{aligned}
$$

ist. Aus Gl. (2) und (3) folgt dann, daß die relative Variation der Mesonenintensität bei einer Temperaturänderung $\delta t$ beträgt

$$
R(p)=\frac{\delta S}{S}=\frac{{ }_{0}^{\int_{B}} e^{F} \delta F d \sigma_{e}}{\int_{0}^{\sigma_{B}} e^{F} d \sigma_{e}} .
$$

Über die Temperaturschwankung wurde angenommen, daß bei einer Veränderung der Intensität der Ultraviolettstrahlung

$$
\delta t=\varepsilon\left(t-t_{0}\right)
$$

wird, d.h. die Temperaturvariation ist proportional der Erwärmung, die durch die Ultraviolettabsorption verursacht wurde.

44 H. E u le r u. W. H e is e n berg, Ergebn. exakt. Naturwiss. XVII, 1 [1938].
Wenn auch über den allgemeinen Gang der Temperatur in der oberen Ozonosphäre heute wohl kein Zweifel mehr bestehen kann, der genaue Verlauf im Einzelfall darf noch recht verschieden sein und $u$. a. wesentlich von dem Ort und der Zeit der Beobachtung abhängen. Für die numerische Auswertung haben wir deshalb zwei verschiedene Rechnungen durchgeführt.

In dem ersten Fall wurde ein Temperaturverlauf entsprechend der in Abb. 1 als ausgezogene Linie eingezeichneten Funktion zugrundegelegt. Die Kreuze und Kreise markieren die Sommer- bzw. Wintertem-

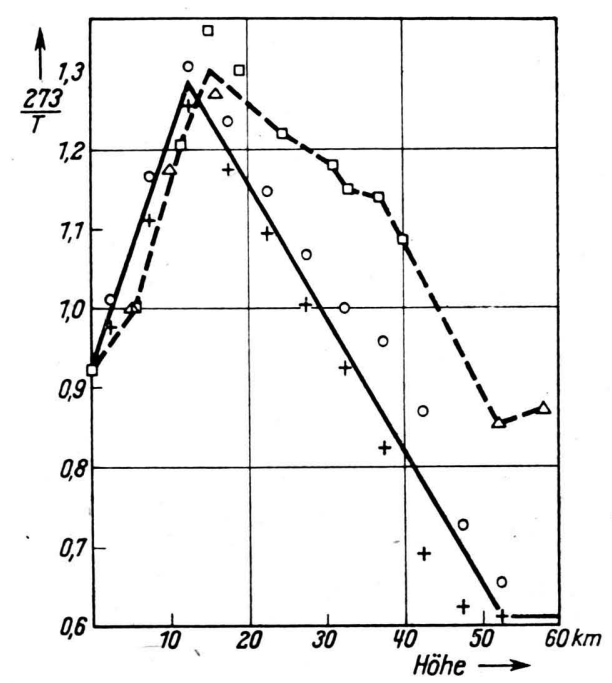

Abb. 1.

peraturen, die von $\mathrm{Gow}$ a ${ }^{42}$ für die Ozonosphäre bei $50^{\circ}$ Breite, $6000^{\circ} \mathrm{K}$ Sonnentemperatur und $0,28 \mathrm{~cm}$ totalem Ozon- und $10 \%$ Wasserdampfgehalt der Atmosphäre berechnet wurden.

Für die mittlere freie Weglänge der Primärteilchen wurde entsprechend dem geometrischen Wirkungsquerschnitt der Luftmoleküle $l=53 \mathrm{~cm} \mathrm{H}_{2} \mathrm{O}$, ferner für $\tau=2 \cdot 10^{-6} \mathrm{sec}, \mu=220 \mathrm{~m}_{\text {e }}$, d. h. $p_{\tau}=1,480 \cdot 10^{9} \mathrm{eV} / c$ und $t_{0}=0,916$ gesetzt.

Die berechneten numerischen Werte der Mesonenintensität $\boldsymbol{S}(p)$ in Seehöhe weichen nur sehr wenig von den Werten ab, die für eine isotherme Atmosphäre von $0^{\circ} \mathrm{C}$ erhalten werden, weil die niedere Temperatur der oberen Troposphäre und unteren Stratosphäre weitgehend durch die Übertemperatur der oberen Ozonosphäre kompensiert werden.

Der Nenner in Gl. (4) läßt sich deshalb gut durch eine $\gamma$-Funktion approximieren, und mit Rücksicht darauf, daß die Temperaturvariation $\delta t$ nur bei 
$\sigma<\sigma_{g} \ll \sigma_{B}$ auftritt *, läßt sich Gl. (4) weiter entwickeln zu:

$$
\begin{aligned}
& R(p)=-\left(\begin{array}{c}
1 \\
(\omega\llcorner 1) ! l
\end{array}\right.
\end{aligned}
$$

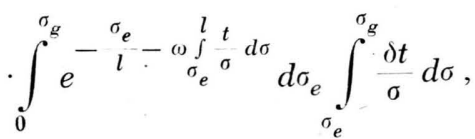

wobei

bezeichnet.

$$
\omega=\frac{p_{\tau}}{q+p}
$$

Die numerische Auswertung ergab, daß bei einer Temperaturvariation von etwa $20^{\circ} \mathrm{C}$ in $40 \mathrm{~km}$ Höhe die Mesonenintensität in Meereshöhe sich um die folgenden relativen Werte ändert:

\begin{tabular}{|c|c|c|c|c|c|c|c|}
\hline in $10^{9} \mathrm{eV} / \mathrm{c}$ & 0 & 1 & 3 & 5 & 10 & 50 & $\infty$ \\
\hline
\end{tabular}

$p$

$R$

in $\% \quad \begin{array}{lllllll}0,22 & 0,25 & 0,27 & 0,22 & 0,14 & 0,04 & 0\end{array}$

Dies ist zu vergleichen mit dem experimentellen Ergebnis, wonach die Amplitude etwa $0,2 \%$ der $\mathrm{Ge}-$ samtintensität ist.

Bei der zweiten Rechnung wurden die der gestrichelten Linie (Abb. 1) entsprechenden Temperaturwerte angewendet. Die Vierecke bedeuten die Werte aus einer Ballonmessung ${ }^{45}$, die Dreiecke Temperaturen, die mit V 2 erhalten wurden ${ }^{41}$. $l$ wurde $\mathrm{zu}$ $60 \mathrm{~cm} \mathrm{H}_{2} \mathrm{O}$ und $t_{0}=0,78$ angenommen.

Unter diesen Voraussetzungen führte die Rechnung zu dem Ergebnis, daß schon $10^{\circ} \mathrm{C}$ Temperaturschwankung in $40 \mathrm{~km}$ Höhe ausreicht, um die folgenden Amplituden in der kosmischen Strahlung zu erzeugen:

\begin{tabular}{lccccccc}
$\begin{array}{l}p \\
\text { in } 10^{9} \mathrm{eV} / c\end{array}$ & 0,5 & 1 & 3 & 5 & 10 & 50 & $\infty$ \\
\hline $\begin{array}{l}R \\
\text { in } \%\end{array}$ & 0,35 & 0,35 & 0,27 & 0,23 & 0,16 & 0,09 & 0
\end{tabular}

Weil in $40 \mathrm{~km}$ Höhè die Erwärmung infolge Ultraviolettabsorption etwa zwischen $80^{\circ}$ und $40^{\circ} \mathrm{C}$ beträgt und nach den vorher berichteten Untersuchungen der veränderliche Anteil der solaren Ultraviolettstrahlung von der gleichen Größenordnung wie der konstante Anteil sein dürfte, kann eine Temperaturschwankung von der berechneten Größenordnung nicht als unplausibel bezeichnet werden.

\footnotetext{
* $\sigma_{g}$ ist die Grenze der Temperaturvariation. Für $\sigma \geq \sigma_{g}$ wird $\delta t=0$.

45 Ch. J. B r a s ef i eld, Physik. Bl. 5, 441 [1949].
}

\section{a) Amplitude \\ 5. Diskussion}

In Anbetracht des angewandten, sehr schematischen Modells für die harte Komponente darf den berechneten Werten nicht eine zu große quantitative Bedeutung zugewiesen werden ${ }^{* *}$.

Bemerkenswert ist der allgemeine Verlauf der Amplitude der 27-tägigen Variation in Abhängigkeit von der Energie der Mesonen, welcher zeigt, daß die Variation bis zu recht hohen Impulsen hinaufreichen kann und u. U., entsprechend der ersten Rechnung, ihr Maximum nicht bei niedrigen Impulsen haben muß.

Die physikalische Ursache dieses Phänomens liegt darin, daß Mesonen mit kleiner Energie wegen ihrer kurzen mittleren Weglänge überwiegend aus niedrigen Schichten kommen, wo keine starken Temperaturschwankungen vorhanden sind; während Mesonen von sehr großer Energie wiederum wegen ihres sehr großen mittleren Zerfallweges nicht mehr durch Verschiebung der Entstehungshöhe merklich beeinflußt werden.

Der spektrale Verlauf ist vereinbar mit der Tatsache, daß durch stärkere Abschirmung der Ionisationskammer die Amplitude sich nicht meßbar ändert.

Die von Broxon untersuchten kleinen Stöße werden wahrscheinlich nicht durch $\mu$-Mesonen ausgelöst. Dieses zeigt ihr besonders großer Barometereffekt ${ }^{13}$. Aus der großen Amplitude ihrer 27-Tage-Variation kann gefolgert werden, daß sie durch Teilchen, die eine kürzere Lebensdauer haben als die $\mu$-Mesonen, erzeugt werden. Auf dieses Problem der stoßauslösenden Primärteilchen werden wir in dem 2. Teil noch ausführlich zurückkommen.

\section{b) Phasenverhältnisse}

Phasenverschiebung zwischen Sonnenfleckenrelativzahlen und kosmischer Strahlung kann auftreten, weil 1. die solare Ultraviolettstrahlung selbst hinter den Sonnenfleckenrelativzahlen zurückbleibt, 2. die Temperatur der Ozonschicht nur träge, mit Verspätung der Schwankung der solaren Ultraviolettstrahlung folgt.

Zwischen der Variation der Sonnenfleckenrelativzahlen und der Ultraviolettstrahlung beträgt die Phasenverschiebung nach $\mathrm{B}$ a r te $1 \mathrm{~s} 27,28$ weniger als

** Insbesondere ist es wahrscheinlich, daß die harte Komponente bei großen Energien die Teilchen enthält, die von $\mu$-Mesonen verschieden sind. Es können in Frage kommen sehr energiereiche $\pi$-Mesonen, neutrale Mesonen, Mesonen von der Masse 1000 oder andere Teilchen. 
einen Tag. K i e pen h e u er findet längere Verspätung, entsprechend der Impulsstärke 1 bis 5 Tage ${ }^{33}$.

Einen Anhalt für die allgemeine Wärmetätigkeit gibt die Berechnung der nächtlichen Abkühlung der oberen Ozonosphäre von Gowan ${ }^{46}$. Nach seinen Ergebnissen ist die nächtliche Abkühlung klein, weniger als $1 / 5$ der Temperaturerhöhung, die infolge der Ultraviolettabsorption vorhanden ist.

Graziade $i^{24}$ fand, daß die 27 -tägige Variation der kosmischen Strahlung eine Phasenverschiebung von etwa 4,5 bis 7,5 Tagen hinter den Sonnenfleckenrelativzahlen zeigt.

In eigenen Untersuchungen fanden wir mit Hilfe der Chree-Methode, daß die kosmische Strahlung im Mittel etwa 4 bis 6 Tage hinter den Sonnenfleckenrelativzahlen zurückbleibt. Allgemein scheint es, daß, je größer der Impuls in den Sonnenfleckenrelativzahlen ist, um so schneller ihm die kosmische Strahlung folgt.

Die Erwärmung und Abkühlung in verschiedenen Höhen der Ozonschicht erfolgt verschieden schnell, es können deshalb Phasenverschiebungen auch zwischen verschiedenen Energiebereichen oder zwischen verschiedenen Komponenten auftreten.

Die Messungen mit Ionisationskammer, wo etwa die gleichen Teilchenarten beobachtet wurden, geben übereinstimmende Werte der Phasenlage ${ }^{3}$. Demgegenüber zeigen Zählrohrregistrierungen der ungefilterten vertikalen Komponente von Kolhörster, die zur selben Zeit wie die Ionisationskammer-Messungen von $\mathrm{W}$ ä $\mathrm{f} f \mathrm{l}$ e r durchgeführt wurden, stärkere Abweichungen in der Phase ${ }^{4 \bar{\tau}}$.

Ebenso ist auch die 27-tägige Variation der kleinen Stöße nicht sehr eng mit der allgemeinen Ionisation korreliert ${ }^{12,13}$.

\section{B. Intensitätsschwankung der kosmischen Strahlung mit dem Sonnenzyklus}

III. Über die Existenz einer 11 - JahrePeriode

Nach den vorhergehenden Untersuchungen kann man erwarten, daß die zyklische Schwankung der Sonnentätigkeit von 11 Jahren Periodenlänge auch in

46 E. H. G o w a n, Proc. Roy. Soc. [London], Ser. A 190, 227 [1947].

47 R. W ä f f l e r, Helv. physica Acta 14, 215 [1941].

* Die erwärmende Wirkung der Ultraviolettstrahlung sollte ferner einen Tagesgang und eine Jahresperiode in der kosmischen Strahlung verursachen. Bei diesen Perioden sind die Verhältnisse jedoch infolge der Mitwirkung anderer Faktoren, deren Anteil sich nur unsicher angeben läßt, wesentlich komplizierter. der Intensität der kosmischen Strahlung in Erscheinung treten wird. Weil die 11-Jahre-Periode der Sonnentätigkeit eine größere Amplitude als die 27Tage-Variation hat, ist es sogar wahrscheinlich, daß hierbei ihr Einfluß noch klarer hervortritt*.

Auf Grund der Annahme, daß die Sonne ständig einen merklichen Anteil der kosmischen Strahlung aussendet, dürfte auch in der kosmischen Strahlung eine 11-Jahre-Periode zu erwarten sein. In diesem Falle soll der Höchstwert der kosmischen Strahlung mit dem Maximum der Sonnentätigkeit und das Minimum der kosmischen Strahlung mit dem Minimum der Sonnentätigkeit zusammenfallen. Die Theorie des magnetischen Ringstromsystems der 27-TageVariation der kosmischen Strahlung ${ }^{2,} 3$ würde ebenfalls eine 11-Jahre-Variation wahrscheinlich machen, jedoch mit negativer Korrelation. Wenn man die 27-Tage-Variation der kosmischen Strahlung auf das allgemeine Magnetfeld der Sonne zurückführt ${ }^{1}$, so wäre keine 11-Jahre-Periode in der kosmischen Strahlung zu erwarten ${ }^{* *}$.

Im folgenden soll der Einfluß des Sonnenzyklus auf die Intensität der kosmischen Strahlung an Hand der Intensitätsregistrierungen von Huancayo und Cheltenham ${ }^{48}$ untersucht werden. Soweit wir übersehen konnten, wurden nur an diesen Stationen genügend lange homogene Messungen durchgeführt und veröffentlicht.

Die geographische Lage von Huancayo ist $12,05^{\circ} \mathrm{S}$; $75,3^{\circ} \mathrm{W} ; 3350 \mathrm{~m}$; die von Cheltenham ist $38,7^{\circ} \mathrm{N}$; $76,8^{\circ} \mathrm{W} ; 72 \mathrm{~m}$. An beiden Stationen wurden Ionisationskammern des Typs „Carnegie Institution's Model C“ verwendet. Die Kammern waren allseitig mit $11,7 \mathrm{~cm} \mathrm{~Pb}$ - ̈̈quivalent abgeschirmt. Die Messung der Höhenstrahlungsintensität erfolgte durch Vergleich der allgemeinen Ionisation mit der Ionisation eines Standard-Uranpräparats. Die Ergebnisse sind in der Form veröffentlicht, daß die Intensität der kosmischen Strahlung durch die Abweichung vom Normalpräparat, ausgedrückt in Prozenten der kosmischen Strahlung, angegeben wird. Die Messungen sind auf Luftdruck und Stöße korrigiert. Eine Korrektion auf Apparaturtemperatur war nicht erforderlich, weil infolge geeigneter Konstruktion die Temperatur der

48 J. L a n g e u. S. E. F o r b u s h, Cosmic-ray results from Huancayo Observatory, Peru, June 1936, Dezember 1946, Carnegie Inst. Wash. Pub. XIV, 175. Washington 1948

** Es sei denn, daß eine 11-Jahre-Variation im allgemeinen Magnetfeld der Sonne angenommen wird. Aber auch diese Annahme würde noch immer zu Widersprüchen mit den experimentellen Ergebnissen führen. 


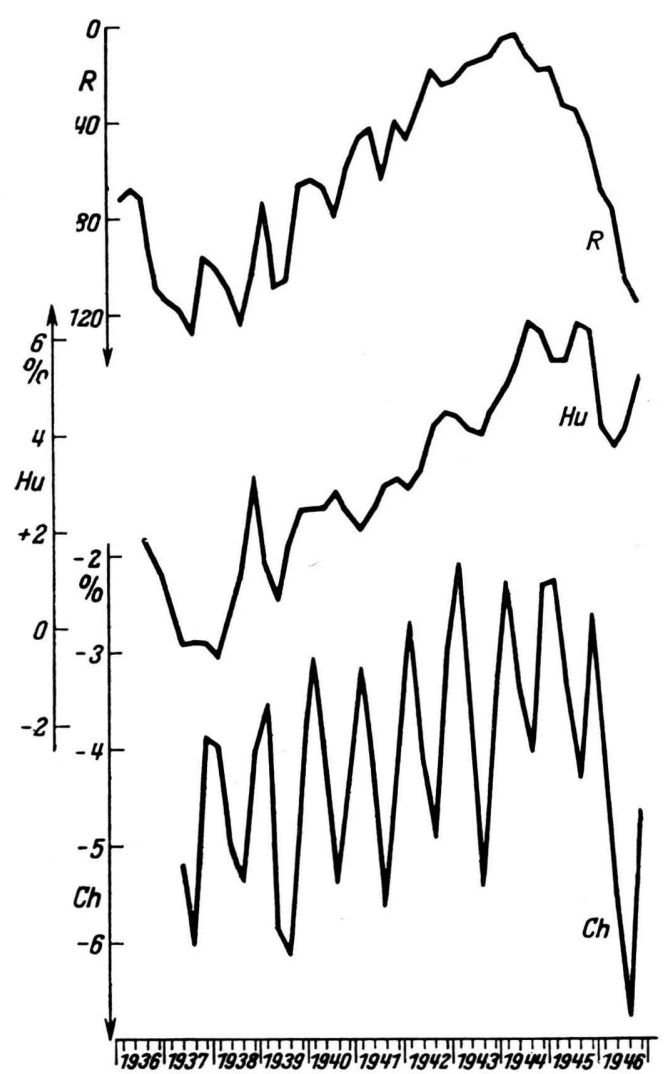

Abb. 2. Dreimonatsmittel der kosmischen Strahlung und der Sonnenfleckenrelativzahlen. Die Kurven sind gekennzeichnet mit $\mathrm{Hu}$ und $\mathrm{Ch}$ entsprechend der Strahlungsintensität in Huancayo bzw. Cheltenham. Die Sonnenfleckenrelativzahlen (R) sind mit nach unten gerichteter Ordinatenachse aufgetragen.

Ionisationskammer ohne Einfluß war. Die Untersuchungen erstreckten sich in Huancayo auf die Zeitspanne von Juni 1936 bis Dezember 1946 und in Cheltenham von März 1937 bis Dezember 1946.

In Abb. 2 wurden die Dreimonatsmittel der kosmischen Strahlung als Funktion der Zeit eingetragen. In den oberen Teil der Zeichnung sind die Dreimonatsmittel der Sonnenfleckenrelativzahlen mit nach unten gerichteter Ordinatenachse eingezeichnet $(\mathrm{R})^{*}$. Alle drei Kurven zeigen einen deutlich parallelen Verlauf. Bei Cheltenham ist der Jahresgang stark ausgeprägt.

Abb. 3 zeigt den Verlauf der Jahresmittel. Die Bedeutung der Ordinaten ist dieselbe wie vorher. Die punktierte bzw. gestrichelte Linie entspricht der

* Es wurden die Züricher Sonnenfleckenrelativzahlen für die ganze Scheibe verwendet, entnommen aus Bulletin for Character Figures of Solar Phenomena.
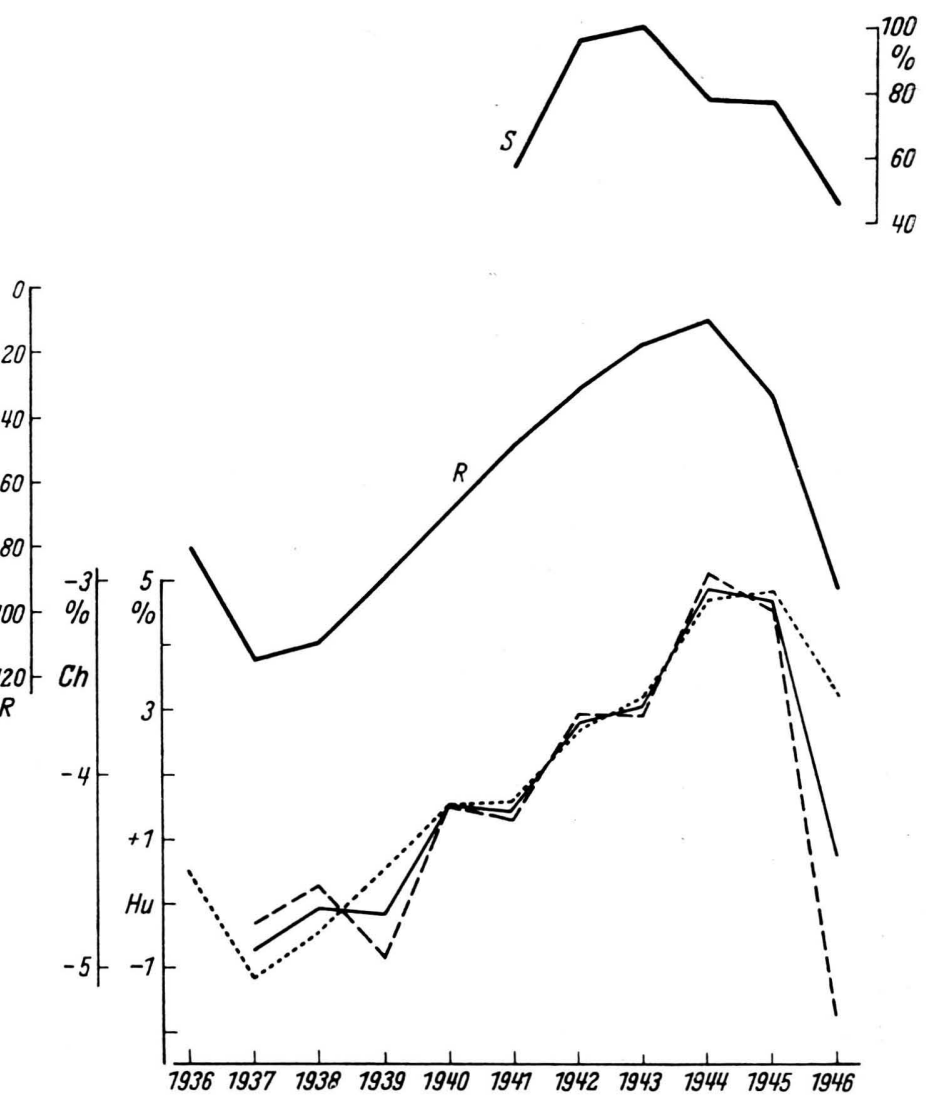

Abb. 3. Jahresmittel der kosmischen Strahlung und der Sonnenfleckenrelativzahlen. Die punktierte bzw. gestrichelte Linie entspricht der Strahlungsintensität in Huancayo bzw. in Cheltenham. Die ausgezogene Linie kennzeichnet den Mittelwert aus beiden Stationen. Die Sonnenfleckenrelativzahlen (R) sind mit nach unten gerichteter Ordinatenachse aufgetragen. Kurve S zeigt die relative Häufigkeit von kleinen Hoffmannschen Stößen in Huancayo. (Zur Auslösung dieser Stöße ist etwa $4 \cdot 10^{10} \mathrm{eV}$ erforderlich.)

Strahlungsintensität in Huancayo bzw. in Christchurch. Die ausgezogene Linie kennzeichnet den Mittelwert aus beiden Stationen. Die Korrelation zwischen den Kurven tritt noch deutlicher hervor und ist als sehr gut zu bezeichnen.

Die Korrelation zwischen den einzelnen Kurven wurde üblicherweise berechnet. Die Fehlergrenzen der Korrelationskoeffizienten wurden nach R. A. Fischer mit Hilfe der Korrelationsziffern bestimmt. Im folgenden stehen rechts und links die Fehlergrenzen entsprechend dem mittleren Fehler der Korrelationsziffern. Die Korrelationskoeffizienten betragen im einzelnen für die 
kosmische Strahlung in Huancayo und Sonnenfleckenrelativzahlen:

$$
r=-0,74 \ldots-0,86 \ldots-0,93,
$$

kosmische Strahlung in Cheltenham und Sonnenfleckenrelativzahlen:

$$
r=-0,78 \ldots-0,89 \ldots-0,95 \text {, }
$$

Mittelwertskurve der kosmischen Strahlung und Sonnenfleckenrelativzahlen:

$$
r=-0,82 \ldots-0,91 \ldots-0,96,
$$

kosmische Strahlung in Huancayo und in Cheltenham:

$$
r=+0,45 \ldots+0,70 \ldots+0,85 \text {. }
$$

Beachtenswert ist ferner der recht große Betrag der Schwankung in der Intensität der kosmischen Strahlung. Die Amplitude in Cheltenham beträgt etwa $2 \%$. Die Intensitätsschwankung in Huancayo hat den Wert von 6\%; es ist jedoch möglich, wie die Betrachtung von Abb. 3 zeigt, daß ein Teil hiervon durch irgendeine systematische Veränderung verursacht wurde.

Abb. 4 zeigt das Punktwolkendiagramm der Jahresmittel; die Intensität der kosmischen Strahlung ist als Funktion der Sonnenfleckenrelativzahlen aufgetragen. Die Pfeile deuten die zeitliche Reihenfolge der einzelnen Meßpunkte an.

Außer der allgemeinen Ionisation wurden auch die Häufigkeit der Ionisationskammer-Stöße, beobachtet im Zeitraum von 1941 bis 1946, veröffentlicht ${ }^{48}$. Die Stöße sind dabei entsprechend der Anzahl der erzeugten Ionen der Größe nach geordnet. Numerisch

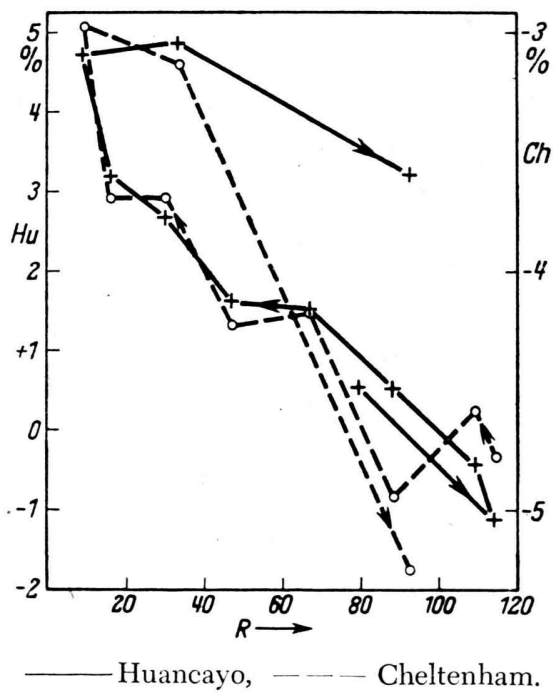

Abb. 4. Die Jahresmittel der kosmischen Strahlung als Funktion der Sonnenfleckenrelativzahlen. Die Pfeile geben die zeitliche Reihenfolge an.

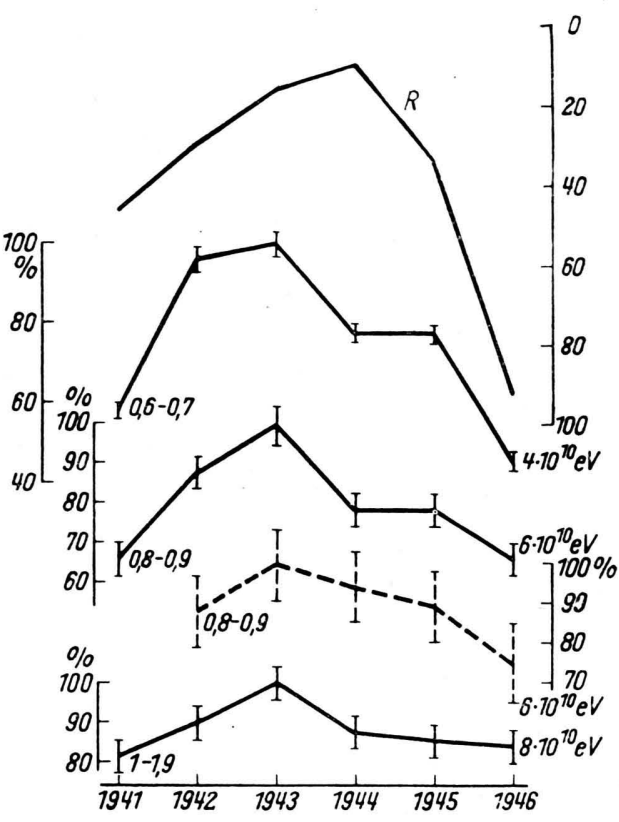

Abb.5. Jahresmittel der Hoffmannschen Stöße in Huancayo (ausgezogene Linie). An der rechten Seite der Kurven sind die mittleren Energien der auslösenden Primärteilchen angegeben. Die Häufigkeit während 1943 wurde gleich 100\% gesetzt. In den Meßpunkten sind die Fehlergrenzen eingezeichnet. Die obere Kurve (R) zeigt die Jahresmittel der Sonnenfleckenrelativzahlen wieder mit nach unten gerichteter Ordinatenachse aufgetragen. Die gestrichelte Linie entspricht einer Häufigkeitskurve in Cheltenham.

wurde diese Anzahl in \% der Ionenmenge, die in 1 Stde. von der allgemeinen Ionisation erzeugt werden, ausgedrückt. Abb. 3 enthält die Häufigkeitskurve der Stöße bei Huancayo von der Stärke $0,6-0,7 \%$ (Kurve S). Hierbei wurde die Häufigkeit während des Jahres 1943 willkürlich gleich 100\% gesetzt. Nach Schein und Gill ${ }^{49}$ sind zur Erzeugung dieser Stöße etwa $4 \cdot 10^{10} \mathrm{eV}$ Energie erforderlich.

In Abb. 5 sind noch die Häufigkeiten der Stoßgrößen von $0,8-0,9 \%$ (mittlere Energie $6 \cdot 10^{10} \mathrm{eV}$ ) und $1,0-1,9 \%$ (mittlere Energie $8 \cdot 10^{10} \mathrm{eV}$ ) eingetragen. Beidemal wurde ebenfalls die Häufigkeit von 1943 gleich 100\% normiert. Die Sonnenfleckenrelativzahlen sind wiederum mit nach unten gerichteter Ordinate aufgetragen *.

Auffallend ist die sehr große Schwankung; bei der Stoßgröße von $0,6 \%$ beträgt die Veränderung in der Häufigkeit $\sim 1: 2$. Mit größeren Energien wird die Amplitude kleiner.

49 M. S chein u. P. S. Gill, Rev. Mod. Physics 11, 267 [1939].

* Die Häufigkeit noch größerer Stöße ist zu klein, um sie in ähnlicher Weise auswerten zu können. 
Von Cheltenham sind die Meßergebnisse nur seit 1942 veröffentlicht; ferner ist die absolute Häufigkeit wesentlich niedriger und der Kurvenverlauf ist statistisch nicht gesichert. Die gestrichelte Linie in Abb. 5 gibt die Stöße der Stärke 0,8—0,9\% wieder.

IV. Theoretische Deutung der 11 - JahrePeriodederkosmischen Strahlung

Die Deutung der 11-Jahre-Periode der allgemeinen Ionisation kann analog zur 27-Tage-Periode erfolgen.

Die starke Schwankung der energiereichen Stöße macht es wiederum unwahrscheinlich, daß es sich um eine magnetische Beeinflussung handelt. Andererseits spricht die negative Korrelation gegen eine solare Komponente als wesentliche Ursache der 11-JahrePeriode. In diesem Falle wäre es auch schwierig, das Auftreten von energiereichen Teilchen mit etwa $10^{11} \mathrm{eV}$ zu erklären.

Das Verhältnis der Amplitude der 27-Tage- und der 11-Jahre-Periode der kosmischen Strahlung beträgt etwa $1: 10$. Wenn wir wieder annehmen, daß diese Schwankungen über die Ultraviolettstrahlung der Sonne und die Temperatur am Ort der Mesonenerzeugung hervorgerufen sind, so dürfte die größere Schwankung bei der 11-Jahre-Periode einerseits auf größere Schwankung der Ultraviolettstrahlung während des Sonnenzyklus zurückzuführen sein ${ }^{* *}$; andererseits kann auch angenommen werden, daß sich die Temperaturschwankung auch auf tiefere atmosphärische Schichten erstrecken wird. Die numerische Abschätzung wurde bei der 27-Tage-Variation dadurch erleichtert, daß die Veränderungen als differentiell angenommen werden konnten. Bei der 11-JahrePeriode kann diese Vereinfachung nicht mehr gemacht werden und wir müssen uns mit der Feststellung begnügen, daß die größere Amplitude der 11-JahrePeriode mit den gemachten Annahmen vereinbar erscheint.

Wesentlich komplizierter sind die Verhältnisse bei den Ionisationskammerstößen. Die Verschiebung der Isobaren, die erforderlich ist, um die Schwankungen zu erklären, wenn die auslösenden Teilchen ebenfalls $\mu$-Mesonen wären, liegt außerhalb des Möglichen. Sie würden etwa um den Faktor $100 \mathrm{zu}$ groß sein. Man weiß jedoch, daß die Stöße durch Teilchen ausgelöst

\footnotetext{
** Die gesamte Schwankung der Sonnenfleckenrelativzahlen in Mittelwertkurve der Sonnenrotationen 17 1433-1439 (gebildet aus Tageswerten) betrug 50; die Schwankung während der untersuchten 11-Jahre-Periode war in Monatsmitteln 165, in Jahresmitteln 105.

50 R. E. L a p p, Physic. Rev. 64, 255 [1943].
}

werden, die - wenigstens zu einem bedeutenden Anteil - nicht $\mu$-Mesonen sind ${ }^{13}{ }^{50}$. Ihre Natur und genauen Eigenschaften sind jedoch nicht bekannt. Es können z. B. - wie schon bei der 27-Tage-Variation erwähnt wurde - energiereiche $\pi$-Mesonen, neutrale Mesonen, Mesonen von der Masse 1000 oder andere Teilchen sein.

In bezug auf energiereiche Protonen, die bei Zählrohrmessungen die durchdringenden Schauer erzeugen und etwa mit dem geometrischen Wirkungsquerschnitt absorbiert werden, sagen George und Trent ${ }^{51}$ : „Penetrating bursts thus show much less of an increase with altitude than do penetrating showers, and we can therefore eliminate the nucleon componente as the primary agent of penetrating bursts."

Christy und Oppenheimer weisen auf Grund allgemeiner Überlegungen über den Verlauf der kosmischen Strahlung in der Atmosphäre darauf hin, daß kurzlebige Mesonen $\left(\sim 10^{-8} \mathrm{sec}\right)$ die stoßauslösenden Teilchen sein könnten.

$\mathrm{L} \mathrm{a} \mathrm{p} \mathrm{p}{ }^{50}$ folgert aus der Analyse der Ionisationskammer-Messungen in Huancayo, daß eine große Anzahl der Stöße entweder durch Luftschauer oder durch Teilchen mit dem Spin 1 ausgelöst wird. In einer späteren Arbeit wurde von $\mathrm{F}$ a hy und $\mathrm{S}$ ch e in ${ }^{53}$ der Anteil der Luftschauer besonders untersucht. Sie haben mit einer durch 7,7, später $12 \mathrm{~cm} \mathrm{~Pb}$ geschirmten Ionisationskammer und mit über die Ionisationskammer gelegten Zählrohren große Stöße in $11200 \mathrm{Fu}$ Höhe (Climax, Colorado) gemessen. Es zeigte sich, daß nur wenige Prozent der Stöße unter Blei von ausgedehnten Luftschauern begleitet waren. Fahy und Schein folgern, daß die Stöße durch Zerfall von Mesonen mit einer Lebensdauer kürzer als $10^{-8} \mathrm{sec}$ erzeugt sein sollten.

Die in Huancayo gefundenen großen Schwankungen lassen sich durch plausible Isobarenverschiebungen erklären, wenn die schauerauslösenden Teilchen etwa von der Lebensdauer $10^{-8}$ sec sind. Ein Meson mit dieser Lebensdauer (und der Masse 300) würde bei einer Energie von $1,5 \cdot 10^{11} \mathrm{eV}$ einen mittleren Zerfallsweg von $3 \mathrm{~km}$ haben. Es reicht dann schon eine Isobarenverschiebung von $3 \mathrm{~km}$ aus, um eine Intensitätsschwankung von etwa $1: 2 \mathrm{zu}$ erzeugen. Mit zunehmender Energie wird der Zerfallsweg

51 E. P. G e orge u. P. T. Tre n t, Nature [London] 161, 248 [1948].

52 R. E. Christy u. J. R. Oppenbeimer, Physic. Rev. 60, 159 [1941].

53 E. F. Fahy u. M. S chein, Physic. Rev. 73, 1227 [1948]. 


\begin{tabular}{|c|c|c|c|c|}
\hline \multirow{2}{*}{ Jahr } & \multicolumn{5}{c}{$\begin{array}{c}\text { S t o } \beta \mathrm{g} \mathrm{r} \text { ö } \mathrm{e} \\
\end{array}$} & $0,6-0,7 \%$ & $0,8-0,9 \%$ & $1,0-1,9 \%$ & $2,0 \%$ \\
\hline \multirow{2}{*}{1942} & 4,9 & 3,5 & 3,0 & 2,3 \\
1943 & 5,6 & 3,5 & 2,9 & 2,3 \\
1944 & 3,6 & 2,9 & 2,6 & 1,9 \\
1945 & 4,1 & 3,1 & 2,3 & 2,2 \\
1946 & 2,3 & 3,1 & 2,0 & 1,8 \\
\hline \multirow{2}{*}{ Mittel } & 4,1 & 3,2 & 2,6 & 2,1 \\
\hline
\end{tabular}

Tab. 1.

länger und sollten die Schwankungen kleiner werden, wie es auch die Meßergebnisse tatsächlich zeigen.

Eine gewisse Kontrolle bietet die Abnahme der Häufigkeit der Stöße zwischen Huancayo (3350 m) und Cheltenham $(72 \mathrm{~m})$. Sie läßt sich mit der Annahme von kurzlebigen Mesonen $\left(\sim 10^{-8} \mathrm{sec}\right)$ als auslösenden Teilchen vereinbaren. In Tab. 1 wurde das Verhältnis der Stoßhäufigkeit von Huancayo und Cheltenham zusammengestellt.

Bei größeren Energien wird das Verhältnis kleiner, wie es den Annahmen entspricht.

Eine weitgehende Identifikation der stoßauslösenden Teilchen kann aus der 11-Jahre-Periode natürlich nicht erwartet werden. Zusammenfassend ergibt sich, $\mathrm{da} ß$ neben dem Barometereffekt und der Intensitätsabhängigkeit von der Höhe auch die mit der Sonnentätigkeit antiparallel verlaufende starke Schwankung der Ionisationskammerstöße darauf hinweist, daß sie, wenigstens teilweise, durch kurzlebige Teilchen (etwa $10^{-8} \mathrm{sec}$ ) erzeugt werden.

Zum Schluß möchte ich Hrn. Prof. W. H e is e n b e r g und Hrn. Prof. L. B i e rm a n n für wertvolle Diskussionen und Förderung dieser Arbeit bestens danken.

\title{
Zur Frage der Existenz neuer ${ }^{a}$-Strahler
}

\author{
Von B. Grsae \\ Aus dem ehemaligen Kaiser-Wilhelm-Institut für Physik, Hechingen \\ (Z. Naturforschg. 5 a, 530-533 [1950]; eingegangen am 8. September 1950)
}

\begin{abstract}
An einem mit Ammoniak gefällten Präparat, das durch wechselweise Fällung mit Flußsäure im oxydierten und reduzierten Zustand gewonnen wurde, wird die Reichweitekurve der $\alpha$-Strahlung gemessen. Danach wird das darin vorhandene Uran durch Auswaschen mit Ammoniumcarbcnat entfernt. Die neue Kurve zeigt die Reichweiten 2,1 cm, 2,5 cm und wahrscheinlich 3,0 cm, die nicht von Uran herrühren. Da mit Flußsäure von den Elementen bis 92 sonst nur Calcium, die Seltenen Erden und Thorium fällbar sind, die sich aber mit Sicherheit ausschließen lassen, müssen diese $\alpha_{-}$-Strahlen einem oder mehreren Elementen größer als 92 zugeschrieben werden. Die Messung der $\beta$-Emission und ihrer Absorption in Aluminium, die mit der der Folgeprodukte von Uran verglichen wird, bestätigt dieses Ergebnis.
\end{abstract}

A uf Grund früher erworbener Kenntnisse einiger chemischer Eigenschaften neuer $\alpha$-Strahler ${ }^{1}$ wurde versucht, durch mehrfache wechselweise Anwendung der Fällung mit Flußsäure, und zwar einmal im mit Ammoniumpersulfat oxydierten Zustand, wobei die gesuchte Substanz im Filtrat bleibt, einmal in Gegenwart von schwefliger Säure, also im reduzierten Zustand, wo eine Fällung auftritt, diese die $\alpha$-Strahlung hervorbringende Substanz möglichst rein darzustellen.

Es ist nun bekannt, daß mit Flußsäure die Seltenen Erden, Thorium, Calcium und 4-wertiges Uran fallen. . Die ersten drei fallen immer, auch wenn zuvor mit Ammoniumpersulfat oxydiert wurde, während die

1 B. Gys a e u. H. Kors ching, Z. Naturforschg. 2a, 475 [1947].
Substanz im Filtrat bleibt; außerdem konnten Thorium und die Seltenen Erden schon vorher weitgehend durch eine Fällung mit Oxalsäure aus sehr schwach saurer Lösung abgetrennt werden. Man kann letztere also mit großer Sicherheit entfernen. Die Abtrennungsmöglichkeit des Thoriums wurde auch dadurch bestätigt, daß solche Präparate auf photographischen Platten, wobei gerade Thorium an dem gleichzeitigen Auftreten der $\alpha$-Strahlung seiner Folgeprodukte leicht $\mathrm{zu}$ erkennen sein müßte, in keinem Fall die sehr charakteristischen sternförmigen Schwärzungen erkennen ließen.

Uran fällt nur im 4-wertigen, dagegen nicht im 6-wertigen Zustand mit Flußsäure. Schon beim Erhitzen in schwefelsaurer Lösung, sicherlich aber nach dem Oxydieren mit Ammoniumpersulfat sollte alles 